\title{
Tail asymptotics of supremum of certain Gaussian processes over threshold dependent random intervals
}

\author{
Krzysztof Dębicki • Enkelejd Hashorva • Lanpeng Ji
}

Received: 18 July 2013 / Revised: 5 March 2014 /

Accepted: 12 March 2014 / Published online: 6 May 2014

(C) Springer Science+Business Media New York 2014

Abstract Let $\{X(t), t \geq 0\}$ be a centered Gaussian process and let $\gamma$ be a nonnegative constant. In this paper we study the asymptotics of $\mathbb{P}\left\{\sup _{t \in\left[0, \mathcal{T} / u^{\gamma}\right]} X(t)>u\right\}$ as $u \rightarrow \infty$, with $\mathcal{T}$ an independent of $X$ non-negative random variable. As an application, we derive the asymptotics of finite-time ruin probability of time-changed fractional Brownian motion risk processes.

Keywords Tail asymptotics · Large deviations · Weibullian tails · Supremum over random intervals · Gaussian process · Fractional Brownian motion · Fractional laplace motion · Gamma process $\cdot$ Ruin probability

AMS 2000 Subject Classifications Primary 60G15 - Secondary 60G70 • 60K30 • $91 \mathrm{~B} 30$

\section{Introduction}

One of the seminal results in the extreme value theory of Gaussian processes concerns the asymptotic behavior of

$$
\mathbb{P}\left\{\sup _{t \in[0, T]} X(t)>u\right\}, \quad u \rightarrow \infty
$$

K. Dȩbicki $(\bowtie)$

Mathematical Institute, University of Wrocław, pl. Grunwaldzki 2/4, 50-384 Wrocław, Poland e-mail: debicki@math.uni.wroc.pl

E. Hashorva $\cdot$ L. Ji

Faculty of Business and Economics (HEC Lausanne), University of Lausanne,

1015 Lausanne, Switzerland 
where $\{X(t), t \geq 0\}$ is a centered Gaussian process with almost surely (a.s.) continuous sample paths, variance function that attains its unique maximum at exactly one point $t_{0} \in[0, T]$ and $T>0$ is deterministic; see the classical monograph Piterbarg (1996), Adler and Taylor (2007) for a complete survey on distributional properties of extremes of Gaussian processes and related topics.

The aim of this paper is to analyze the asymptotics of a counterpart of Eq. 1 in the case of $T$ being an independent of $X$ non-negative random variable, possibly depending on $u$, namely

$$
p(X, \mathcal{T}, \gamma, u):=\mathbb{P}\left\{\sup _{t \in\left[0, \mathcal{T} / u^{\gamma}\right]} X(t)>u\right\}, \quad \text { with } \gamma \geq 0
$$

as $u \rightarrow \infty$.

Let in the following $B_{\alpha}$ denote a fractional Brownian motion (fBm) with Hurst index $\alpha / 2 \in(0,1]$, and let $\{Y(t), t \geq 0\}$ independent of $B_{\alpha}$ be a non-negative, non-decreasing stochastic process.

Apart from theoretical interest in properties of Eq. 2, the motivation to analyze it comes for numerous applications. For instance the calculation of the finite-time ruin probability of the time-changed fractional Brownian motion risk process $\psi_{T}$ defined by

$$
\psi_{T}(u)=\mathbb{P}\left\{\inf _{t \in[0, T]}\left(u+c Y(t)-B_{\alpha}(Y(t))\right)<0\right\}
$$

for some $c>0, T>0, u \geq 0$ is possible using Monte Carlo simulations only when $Y$ is known. In view of our findings, for all large $u$ compact formulas for approximation of $\psi_{T}(u)$ can be given explicitly.

In the context of theoretical actuarial models, $u$ is the so-called initial reserve, $c$ models the premium income rate, and $B_{\alpha}(t)$ represents the total amount up to time $t>0$ of aggregate claims (including fluctuations). The justification for choosing $\mathrm{fBm}$ to model the aggregate claim process comes from Michna (1998), where it is shown that the finite-time ruin probability $\psi_{T}(u)$, with $Y(t)=t$ is a good approximation of the finite-time ruin probability for the classical risk process with claims possessing long range dependence property. The role of the random process $Y$ is crucial in order to make such models adequate for applications. It is a substitute for the real time, where $Y(t)$ stands for the random business time, which is consistent with the insurance practice where both claims and premiums may not be received immediately at time $t$ of the event, but at a later random time modeled by $Y(t)$. Indeed, if $\{Y(t), t \geq 0\}$ has additionally a.s. continuous sample paths, then re-writing (3) as

$$
\psi_{T}(u)=\mathbb{P}\left\{\sup _{t \in[0, \mathcal{T} / u]} \frac{B_{\alpha}(t)}{1+c t}>u^{1-\alpha / 2}\right\}, \quad \mathcal{T}=Y(T)
$$

we see that the asymptotic analysis of $\psi_{T}(u)$ reduces to the analysis of the asymptotic tail behaviour of supremum of a specific Gaussian process over a random interval.

Other branch of motivations to analyze (2) stems from recently studied problems in fluid queueing theory. In particular, the tail asymptotics of the stationary buffer content of a hybrid fluid queue, with input modelled by a superposition of integrated 
alternating on-off process and a Gaussian process with stationary increments, reduces (under some assumptions) to Eq. 2 with suitably chosen random $\mathcal{T}$; see e.g., Zwart et al. (2005).

In the case that $\{Y(t), t \geq 0\}$ is a Gamma process and $c=0$, investigation of $\psi_{T}(u)$, as $u \rightarrow \infty$ reduces to the analysis of the tail asymptotics of the fractional Laplace motion; see Kozubowski et al. (2006), Arendarczyk and Dȩbicki (2011). For the related literature on time-changed models we refer to Fotopoulos and Luo (2011), who considered the case of Brownian motion $(\alpha=1)$ and $\mathrm{Wu}$ and Wang (2012), where a model based on the Cox risk process, which is a time-changed compound Poisson risk process, is considered. For more applications in finance and insurance, see e.g., Geman et al. (2001) and Palmowski and Zwart (2007), among many others.

Contribution : a) In Theorem 3.1, under some canonical asymptotic assumptions of the Gaussian process $\{X(t), t \geq 0\}$, see Section 2, if $\mathcal{T}$ has a sufficiently heavy tail distribution (which is manifested by insensitivity property of the tail distribution of $\mathcal{T})$, we derive exact asymptotics of $p(X, \mathcal{T}, \gamma, u)$.

b) Theorem 3.3 deals with a more general class of random variables $\mathcal{T}$. Under a log-power tail asymptotic assumption (see Eq. 6), we obtain the logarithmic asymptotics of $p(X, \mathcal{T}, \gamma, u)$. It appears that, depending on the interplay between heaviness of $\mathcal{T}$ and local properties of the variance function of $X$ at 0 we can distinguish four scenarios, leading to four qualitatively different asymptotics.

Our novel result complement recent findings of Arendarczyk and Debicki (2011, 2012) and Tan and Hashorva (2013), where extremes over a random-time interval were analyzed for stationary centered Gaussian processes and centered Gaussian processes with stationary increments respectively. We refer to Zwart et al. (2005), Dębicki and van Uitert (2006), Palmowski and Zwart (2007) for other related results.

The organization of the paper is as follows: In Section 2 we introduce some notation and formulate the main assumptions imposed to the Gaussian process $\{X(t)$, $t \geq 0\}$. Section 3 presents the main results. In Section 4 we discuss the finite-time ruin probability of the time-changed $\mathrm{fBm}$ risk processes. The proofs of all the results are relegated to Section 5 .

\section{Preliminaries}

Throughout this paper $\Psi(\cdot)$ denotes the survival function of a standard Gaussian random variable $N(0,1)$. For two positive functions $a_{1}(\cdot), a_{2}(\cdot)$ on $[0, \infty)$ we write $a_{1}(u) \sim a_{2}(u)$ if $\lim _{u \rightarrow \infty} a_{1}(u) / a_{2}(u)=1$ and $a_{1}(u) \stackrel{\log }{\sim} a_{2}(u)$ if $\lim _{u \rightarrow \infty} \log \left(a_{1}(u)\right) / \log \left(a_{2}(u)\right)=1$. In the sequel $\Gamma(\cdot)$ denotes the Euler Gamma function.

Following, e.g., Foss et al. (2013) we say that a non-negative random variable $\mathcal{T}$ is $h$-insensitive if

$$
\mathbb{P}\{\mathcal{T}>u \pm h(u)\} \sim \mathbb{P}\{\mathcal{T}>u\}
$$

as $u \rightarrow \infty$ for some function $h(\cdot)$. Our first main result in this paper is the exact 
asymptotic behaviour of $p(X, \mathcal{T}, \gamma, u)$ defined in Eq. 2 for $\mathcal{T}$ being $h$-insensitive. Two large classes of distributions for $\mathcal{T}$ that satisfy the insensitivity criteria are:

i) $\mathcal{T}$ is regularly varying at $\infty$ with index $\lambda>0$, which means that $\mathbb{P}\{\mathcal{T}>u\}=$ $\mathcal{L}(u) u^{-\lambda}$, where $\mathcal{L}(\cdot)$ is slowly varying at $\infty$, i.e., for any $x>0$ we have $\lim _{u \rightarrow \infty} \mathcal{L}(x u) / \mathcal{L}(u)=1$ (see e.g., Resnick 1987);

ii) $\mathcal{T}$ is asymptotically Weibullian, i.e.,

$$
\mathbb{P}\{\mathcal{T}>u\} \sim \mathcal{L}(u) u^{\delta} \exp \left(-L u^{p}\right)
$$

as $u \rightarrow \infty$, where $L, p>0$ and $\delta \in \mathbb{R}$ and $\mathcal{L}(\cdot)$ is slowly varying at $\infty$; we abbreviate Eq. 5 as $\mathcal{T} \in \mathcal{W}(p, L, \mathcal{L}, \delta)$.

A significant relaxation of the Weibullian-tail assumption (5) for $\mathcal{T}$ is that it has asymptotically a $\log$-power tail with coefficient $L>0$ and power $p>0$, i.e.,

$$
\lim _{u \rightarrow \infty} \frac{\log \mathbb{P}\{\mathcal{T}>u\}}{u^{p}}=-L .
$$

As above, hereafter $B_{\alpha}$ stands for a fBm with Hurst index $\alpha / 2 \in(0,1]$, i.e., a centered Gaussian process with stationary increments, continuous sample paths a.s. and variance function $\sigma_{B_{\alpha}}^{2}(t)=t^{\alpha}$. Following Piterbarg (1996), we introduce two key constants, namely Pickands constant

$$
\mathcal{H}_{\alpha}:=\lim _{S \rightarrow \infty} S^{-1} \mathbb{E}\left(\exp \left(\sup _{t \in[0, S]}\left(\sqrt{2} B_{\alpha}(t)-t^{\alpha}\right)\right)\right) \in(0, \infty)
$$

and Piterbarg constant

$$
\mathcal{P}_{\alpha}^{R}:=\lim _{S \rightarrow \infty} \mathbb{E}\left(\exp \left(\sup _{t \in[-S, S]}\left(\sqrt{2} B_{\alpha}(t)-(1+R)|t|^{\alpha}\right)\right)\right) \in(0, \infty), \quad R \in(0, \infty) .
$$

In this paper we tacitly assume that $\{X(t), t \geq 0\}$ is a centered Gaussian process with a.s. continuous and bounded sample paths and $\sigma^{2}(t):=\operatorname{Var}(X(t))$ that attains its maximum over $[0, \infty)$ at the unique point $t=t_{0}>0$ with $\sigma\left(t_{0}\right)=1$. Additionally we suppose that:

A1. There exist some positive constants $a, \beta$ such that

$$
\sigma(t)=1-a\left|t-t_{0}\right|^{\beta}+o\left(\left|t-t_{0}\right|^{\beta}\right), \quad t \rightarrow t_{0} .
$$

A2. There exist $d>0, \alpha \in(0,2]$ such that

$$
\operatorname{Cov}\left(\frac{X(s)}{\sigma(s)}, \frac{X(t)}{\sigma(t)}\right)=1-d|t-s|^{\alpha}+o\left(|t-s|^{\alpha}\right), \quad s \rightarrow t_{0}, t \rightarrow t_{0} .
$$

A3. There exist constants $\mathbb{Q}>0, H>t_{0}$ and $r \in(0,2]$ such that, for all $s, t \in$ $[0, H]$ with $|s-t|<1$

$$
\mathbb{E}\left((X(t)-X(s))^{2}\right) \leq \mathbb{Q}|t-s|^{r} .
$$

A4. $\quad \limsup _{t \rightarrow \infty} \sigma(t)<1$. 
We conclude this section with a preliminary result, which gives the exact asymptotics of the supremum of a Gaussian process over a deterministic time interval; see e.g., Piterbarg (1996).

Theorem 2.1 If the assumptions A1-A4 are satisfied, then for any $T>t_{0}$ we have

$$
\mathbb{P}\left\{\sup _{t \in[0, \infty)} X(t)>u\right\} \sim \mathbb{P}\left\{\sup _{t \in[0, T]} X(t)>u\right\} \sim \mathcal{C}_{\alpha, \beta} \Lambda_{\alpha, \beta}(u) \Psi(u), \quad u \rightarrow \infty,(9)
$$

where

$\mathcal{C}_{\alpha, \beta}=\left\{\begin{array}{ll}2 \mathcal{H}_{\alpha} \Gamma(1 / \beta+1) d^{1 / \alpha} a^{-1 / \beta}, & \text { if } \alpha<\beta, \\ \mathcal{P}_{\alpha}^{a / d}, & \text { if } \alpha=\beta, \\ 1, & \text { if } \alpha>\beta,\end{array}\right.$ and $\quad \Lambda_{\alpha, \beta}(u)= \begin{cases}u^{2 / \alpha-2 / \beta}, & \text { if } \alpha<\beta, \\ 1, & \text { if } \alpha \geq \beta .\end{cases}$

Remark The exact asymptotics for the infinite-time interval case can be obtained by a direct application of the Borell-TIS inequality (e.g., Adler 1990 or Adler and Taylor 2007) using further the result for the asymptotics of the supremum of $X$ over any finite-time interval $[0 . T]$.

\section{Main results}

In this section we present our main results. We begin with the derivation of the exact asymptotic behaviour of $p(X, \mathcal{T}, \gamma, u)$ as $u \rightarrow \infty$, which is presented in Theorem 3.1. Then, under milder assumptions on $\mathcal{T}$, we provide a complete study of the logarithmic asymptotics of $p(X, \mathcal{T}, \gamma, u)$ as $u \rightarrow \infty$, see Theorem 3.3.

Theorem 3.1 Let $\{X(t), t \geq 0\}$ be a centered Gaussian process such that assumptions A1-A4 are satisfied, and let $\mathcal{T}$ be a non-negative random variable independent of the Gaussian process $X$.

i) If $\gamma=0$ and $\mathbb{P}\left\{\mathcal{T} \geq t_{0}\right\}>0$, then

$$
p(X, \mathcal{T}, \gamma, u) \sim \mathbb{P}\left\{\mathcal{T} \geq t_{0}\right\} .
$$

ii) If $\gamma>0$ and $\mathcal{T}$ is $u^{1-2 /(\gamma(1+\beta))}$-insensitive, then

$$
p(X, \mathcal{T}, \gamma, u) \sim \mathcal{C}_{\alpha, \beta} \Lambda_{\alpha, \beta}(u) \Psi(u) \mathbb{P}\left\{\mathcal{T} \geq t_{0} u^{\gamma}\right\}
$$

The proof of Theorem 3.1 is given in Section 5.1.

Theorem 3.1 complements recent results of Arendarczyk and Dȩbicki (2011, 2012) and Tan and Hashorva (2013), where the class of centered stationary Gaussian processes and Gaussian processes with stationary increments was analyzed.

As a straightforward consequence of Theorem 3.1 we obtain the following results.

Corollary 3.2 Suppose that the assumptions of Theorem 3.1 are satisfied and that $\gamma>0$.

i) If $\mathcal{T}$ is regularly varying at $\infty$ with index $\lambda>0$, then

$$
p(X, \mathcal{T}, \gamma, u) \sim \mathcal{C}_{\alpha, \beta} \Lambda_{\alpha, \beta}(u) \Psi(u) \mathbb{P}\left\{\mathcal{T} \geq t_{0} u^{\gamma}\right\} .
$$


ii) If $\mathcal{T} \in \mathcal{W}(p, L, \mathcal{L}, \delta)$ with $p \in\left(0, \frac{2}{\gamma(1+\beta)}\right)$, then Eq. 12 holds.

Complementary to the above exact asymptotics, in the next theorem we derive the logarithmic asymptotic behaviour of Eq. 2 for a class of log-power tailed random variables $\mathcal{T}$.

Let us introduce the following notation

$$
\widehat{\sigma}(s):=\sup _{t \in[0, s]} \sigma(t), \quad \widetilde{\sigma}_{L, \gamma}(s):=\frac{1}{2 \widehat{\sigma}^{2}(s)}+L s^{2 / \gamma}, \quad s \geq 0
$$

and

$$
A_{0}=\inf \left\{A: A=\arg \inf _{t \leq t_{0}}\left(\widetilde{\sigma}_{L, \gamma}(t)\right)\right\} .
$$

Theorem 3.3 Let $\{X(t), t \geq 0\}$ be a centered Gaussian process such that assumptions A1-A4 are satisfied and let $\mathcal{T}$ be a non-negative random variable independent of the Gaussian process $X$ with asymptotically log-power tail with coefficient $L>0$ and power $p>0$.

i) If $\gamma p<2$, then

$$
\lim _{u \rightarrow \infty} \frac{\log p(X, \mathcal{T}, \gamma, u)}{u^{2}}=-\frac{1}{2} .
$$

ii) If $\gamma p=2, A_{0}>0$, and on any compact subset of $(0, \infty) \widehat{\sigma}(s)$ is differentiable and $\left|\widehat{\sigma}^{\prime}(s)\right|$ is uniformly bounded, then

$$
\lim _{u \rightarrow \infty} \frac{\log p(X, \mathcal{T}, \gamma, u)}{u^{2}}=-\widetilde{\sigma}_{L, \gamma}\left(A_{0}\right) .
$$

iii) If $\gamma p>2$ and $\sigma(0)>0$, then

$$
\lim _{u \rightarrow \infty} \frac{\log p(X, \mathcal{T}, \gamma, u)}{u^{2}}=-\frac{1}{2 \sigma^{2}(0)} .
$$

iv) If $\gamma p>2$ and $\sigma(t)=D t^{\eta}(1+o(1))$ as $t \downarrow 0$ for some positive constants $D$ and $\eta$, then

$$
\lim _{u \rightarrow \infty} \frac{\log p(X, \mathcal{T}, \gamma, u)}{u^{2 p(\eta \gamma+1) /(2 \eta+p)}}=-A_{1},
$$

where $A_{1}=\frac{1}{2} D^{-\frac{2 p}{2 \eta+p}}(L p / \eta)^{\frac{2 \eta}{2 \eta+p}}+L^{\frac{2 \eta}{2 \eta+p}}\left(\eta /\left(p D^{2}\right)\right)^{\frac{p}{2 \eta+p}}$.

A complete proof of Theorem 3.3 is given in Section 5.2.

Remark It follows straightforwardly from the proof of Theorem 3.3 that statements i) and $i$ ii $)$ also hold if $-\log \mathbb{P}\{\mathcal{T}>u\}=\mathcal{L}(u) u^{p}$ with some slowly varying function $\mathcal{L}(\cdot)$ at infinity.

When $p \gamma=2$ we imposed the assumption that $A_{0}>0$. The special case $A_{0}=$ 0 , which is also possible, is much more involved, and will therefore be considered elsewhere. 


\section{Ruin probability of the time-changed fBm risk processes}

Consider an extension of the time-changed fBm risk process defined in the Introduction, by allowing a power trend-function; i.e., let

$$
Z(t):=u+c(Y(t))^{\theta}-B_{\alpha}(Y(t)), \quad t \geq 0,
$$

where $u \geq 0, c>0, \theta>\alpha / 2$ and $\{Y(t), t \geq 0\}$ is a non-negative non-decreasing stochastic process being independent of $\left\{B_{\alpha}(t), t \geq 0\right\}$. Clearly, $\theta=1$ is a choice leading to our risk model in the Introduction. Related risk models were studied for instance in Dębicki and Rolski (2002), where the finite-time ruin probability with the choice $Y(t) \equiv t$ was analyzed, whereas the infinite-time ruin counterpart was considered in Hüsler and Piterbarg (1999), see also the recent contribution Hashorva et al. (2013).

As in the Introduction the finite-time ruin probability for the risk process (19) is given by

$$
\begin{aligned}
\psi_{T}(u) & =\mathbb{P}\left\{\inf _{t \in[0, T]}\left(u+c(Y(t))^{\theta}-B_{\alpha}(Y(t))\right)<0\right\} \\
& =\mathbb{P}\left\{\sup _{t \in[0, T]}\left(B_{\alpha}(Y(t))-c(Y(t))^{\theta}\right)>u\right\},
\end{aligned}
$$

with $T>0$ and $u \geq 0$.

\subsection{Continuous time-process $Y$}

In this subsection, we apply the results of Theorems 3.1 and 3.3 to the analysis of the asymptotics of the finite-time ruin probability of the time-changed $\mathrm{fBm}$ risk process (19) as $u \rightarrow \infty$, where the time process $Y$ has a.s. continuous sample paths.

Before stating our results for this risk model we need to introduce the following notation

$$
\begin{aligned}
Q & :=2^{\frac{1}{2}+\frac{1}{\alpha}} \sqrt{\pi} c^{\frac{2-\alpha}{2 \theta}} \alpha^{\frac{\alpha-2-\theta}{2 \theta}} \theta^{\frac{2-\alpha}{\alpha}}(2 \theta-\alpha)^{\frac{\theta \alpha-4 \theta+2 \alpha-\alpha^{2}}{2 \theta \alpha}} \\
s_{0} & :=\left(\frac{\alpha}{c(2 \theta-\alpha)}\right)^{1 / \theta}, \quad V_{0}:=\frac{2 \theta-\alpha}{2 \theta} s_{0}^{\alpha / 2}=\frac{2 \theta-\alpha}{2 \theta}\left(\frac{\alpha}{c(2 \theta-\alpha)}\right)^{\frac{\alpha}{2 \theta}} .
\end{aligned}
$$

The main results are presented in Propositions 4.1 and 4.2; their proofs are relegated to Section 5.3.

Proposition 4.1 Assume that $\theta>\alpha / 2, c>0$ and $\{Y(t), t \geq 0\}$ has a.s. continuous sample paths.

i) If $Y(T)$ is regularly varying at $\infty$ with index $\lambda>0$, i.e., $\mathbb{P}\{Y(T)>u\}=$ $\mathcal{L}(u) u^{-\lambda}$, then

$$
\psi_{T}(u) \sim Q \mathcal{H}_{\alpha} s_{0}^{-\lambda} u^{\frac{(2 \theta-\alpha)(2-\alpha)-2 \lambda \alpha}{2 \theta \alpha}} \mathcal{L}\left(s_{0} u^{\frac{1}{\theta}}\right) \Psi\left(V_{0}^{-1} u^{\frac{2 \theta-\alpha}{2 \theta}}\right) .
$$


ii) If $Y(T) \in \mathcal{W}(p, L, \mathcal{L}, \delta)$ with $p \in\left(0, \frac{2 \theta-\alpha}{3}\right), L>0$, and $\delta \in \mathbb{R}$, then

$$
\psi_{T}(u) \sim Q \mathcal{H}_{\alpha} \mathcal{L}\left(s_{0} u^{1 / \theta}\right) s_{0}^{\delta} u^{\frac{(2 \theta-\alpha)(2-\alpha)+2 \delta \alpha}{2 \theta \alpha}} \exp \left(-L s_{0}^{p} u^{\frac{p}{\theta}}\right) \Psi\left(V_{0}^{-1} u^{\frac{2 \theta-\alpha}{2 \theta}}\right) .
$$

Proposition 4.2 Under the setup of Proposition 4.1, suppose further that $Y(T)$ has asymptotically log-power tail with coefficient $L>0$ and power $p>0$.

i) If $2 \theta-\alpha>p$, then

$$
\lim _{u \rightarrow \infty} \frac{\log \psi_{T}(u)}{u^{\frac{2 \theta-\alpha}{\theta}}}=\frac{-1}{2 V_{0}^{2}}
$$

ii) If $2 \theta-\alpha<p$, then

$$
\lim _{u \rightarrow \infty} \frac{\log \psi_{T}(u)}{u^{\frac{2 p}{\alpha+p}}}=-\left(\frac{1}{2}\left(\frac{2 p}{\alpha}\right)^{\frac{\alpha}{\alpha+p}}+\left(\frac{\alpha}{2 p}\right)^{\frac{p}{\alpha+p}}\right) L^{\frac{\alpha}{\alpha+p}}
$$

iii) If $2 \theta-\alpha=p$, then

$$
\lim _{u \rightarrow \infty} \frac{\log \psi_{T}(u)}{u^{\frac{2 \theta-\alpha}{\theta}}}=-\left(\frac{\left(1+c A_{0}^{\theta}\right)^{2}}{2 A_{0}^{\alpha}}+L A_{0}^{2 \theta-\alpha}\right),
$$

where

$$
A_{0}=\left(\frac{c(\alpha-\theta)+\sqrt{c^{2}(\theta-\alpha)^{2}+2 \alpha\left(c^{2}(\theta-\alpha / 2)+L(2 \theta-\alpha)\right)}}{c^{2}(2 \theta-\alpha)+2 L(2 \theta-\alpha)}\right)^{1 / \theta}
$$

\subsection{Discontinuous time-process $Y$}

In several models the time-process $Y$ has discontinuous sample paths. Therefore, in this section we investigate additional cases relaxing the assumption on continuity of sample paths of $Y$.

Proposition 4.3 Assume that $\theta>\alpha / 2, c>0$ and the random variable $Y(T)$ possesses an absolutely continuous distribution with probability density function which is regularly varying at $\infty$ with index $\lambda+1>1$. Then

$$
\lim _{u \rightarrow \infty} \frac{\log \psi_{T}(u)}{u^{\frac{2 \theta-\alpha}{\theta}}}=\frac{-1}{2 V_{0}^{2}}
$$

Proposition 4.4 Assume that $\theta>\alpha / 2, c>0$ and $Y(T)$ possesses absolutely continuous distribution with probability density function $\rho_{Y(T)}(\cdot)$ such that $\lim _{u \rightarrow \infty} \log \left(\rho_{Y(T)}(u)\right) / u^{p}=-L$ for some $p, L>0$.

i) If $2 \theta-\alpha>p$, then

$$
\lim _{u \rightarrow \infty} \frac{\log \psi_{T}(u)}{u^{\frac{2 \theta-\alpha}{\theta}}}=\frac{-1}{2 V_{0}^{2}}
$$


ii) If $2 \theta-\alpha<p$, then

$$
\lim _{u \rightarrow \infty} \frac{\log \psi_{T}(u)}{u^{\frac{2 p}{\alpha+p}}}=-\left(\frac{1}{2}\left(\frac{2 p}{\alpha}\right)^{\frac{\alpha}{\alpha+p}}+\left(\frac{\alpha}{2 p}\right)^{\frac{p}{\alpha+p}}\right) L^{\frac{\alpha}{\alpha+p}} .
$$

iii) If $2 \theta-\alpha=p$, then

$$
\lim _{u \rightarrow \infty} \frac{\log \psi_{T}(u)}{u^{\frac{2 \theta-\alpha}{\theta}}}=-\left(\frac{\left(1+c A_{0}^{\theta}\right)^{2}}{2 A_{0}^{\alpha}}+L A_{0}^{2 \theta-\alpha}\right),
$$

where

$$
A_{0}=\left(\frac{c(\alpha-\theta)+\sqrt{c^{2}(\theta-\alpha)^{2}+2 \alpha\left(c^{2}(\theta-\alpha / 2)+L(2 \theta-\alpha)\right)}}{c^{2}(2 \theta-\alpha)+2 L(2 \theta-\alpha)}\right)^{1 / \theta} .
$$

Example 4.5 Assume that $Y(t)=\sum_{i=1}^{N(t)} Z_{i}$ is a compound Poisson process with $Z_{i}, i \geq 1$ non-negative independent random variables with common probability density function $h(\cdot)$ which is regularly varying at $\infty$ with index $\lambda+1>1$. If $h(\cdot)$ is monotone and $\{N(t), t \geq 0\}$ is a homogeneous Poisson process with intensity $\mu>0$, then by Proposition 4.3

$$
\lim _{u \rightarrow \infty} \frac{\log \psi_{T}(u)}{u^{\frac{2 \theta-\alpha}{\theta}}}=\frac{-1}{2 V_{0}^{2}}
$$

Example 4.6 Let $\left\{\Gamma_{t}, t \geq 0\right\}$ be a Gamma process with parameter $v>0$, i.e., a Lévy process such that the increments $\Gamma_{t+s}-\Gamma_{t}$ have Gamma distribution with probability density function

$$
f(x)=\frac{1}{\Gamma\left(\frac{s}{v}\right)} x^{\frac{s}{v}-1} \exp (-x), \quad x>0 .
$$

By fractional Laplace motion $\left\{L_{\alpha}(t), t \geq 0\right\}$ we denote a random process defined as

$$
\left\{L_{\alpha}(t), t \geq 0\right\} \stackrel{d}{=}\left\{\sigma B_{\alpha}\left(\Gamma_{t}\right), t \geq 0\right\}, \quad \sigma>0 .
$$

A standard fractional Laplace motion corresponds to $\sigma=v=1$; see, e.g., Kozubowski et al. (2004, 2006). Choosing $Y(t)=\Gamma_{t}$, we consider below finite-time ruin probability of risk process modelled by fractional Laplace motion

$\psi_{T}(u)=\mathbb{P}\left\{\inf _{t \in[0, T]}\left(u+c \Gamma_{t}-B_{\alpha}\left(\Gamma_{t}\right)\right)<0\right\}=\mathbb{P}\left\{\sup _{t \in[0, T]}\left(B_{\alpha}\left(\Gamma_{t}\right)-c \Gamma_{t}\right)>u\right\}$.

For this model Proposition 4.4 implies:

i) If $\alpha<1$, then

$$
\lim _{u \rightarrow \infty} \frac{\log \psi_{T}(u)}{u^{2-\alpha}}=\frac{-2}{(2-\alpha)^{2}}\left(\frac{c(2-\alpha)}{\alpha}\right)^{\alpha} .
$$


ii) If $1<\alpha<2$, then

$$
\lim _{u \rightarrow \infty} \frac{\log \psi_{T}(u)}{u^{\frac{2}{\alpha+1}}}=-\frac{1}{2}\left(\frac{2}{\alpha}\right)^{\frac{\alpha}{\alpha+1}}-\left(\frac{\alpha}{2}\right)^{\frac{1}{\alpha+1}} .
$$

iii) If $\alpha=1$, then

$$
\lim _{u \rightarrow \infty} \frac{\log \psi_{T}(u)}{u}=-\frac{\left(1+c A_{0}\right)^{2}}{2 A_{0}}-A_{0},
$$

where $A_{0}=\frac{1}{\sqrt{c^{2}+2}}$.

\section{Proofs}

This section is dedicated to proofs of our results. In what follows, the positive constant $\mathbb{Q}$ may be different from line to line. We begin with a lemma which is of some interests on its own.

Lemma 5.1 Let $X$ be a non-negative random variable which is $u^{1-p}$-insensitive, with $p>0$. Then, for any positive constant $B$

$$
\lim _{u \rightarrow \infty} \frac{\exp \left(-B u^{p}\right)}{\mathbb{P}\{X>u\}}=0 .
$$

Proof of Lemma 5.1 First observe that, for any sufficiently large $u$ there exists some $\theta_{u} \in[0,1]$ such that (set next $Y=X^{p}$ )

$$
\begin{aligned}
\mathbb{P}\{Y>u\} & \leq \mathbb{P}\{Y>u-1\} \\
& =\mathbb{P}\left\{X>(u-1)^{1 / p}\right\} \\
& =\mathbb{P}\left\{X>u^{1 / p}-(1 / p)\left(u-\theta_{u}\right)^{1 / p-1}\right\} \\
& \leq \mathbb{P}\left\{X>u^{1 / p}-(2 / p)(u)^{1 / p-1}\right\} .
\end{aligned}
$$

By insensitivity of $X$, we immediately get that

$$
\mathbb{P}\left\{X>u^{1 / p}-(2 / p)(u)^{1 / p-1}\right\} \sim \mathbb{P}\left\{X>u^{1 / p}\right\}=\mathbb{P}\{Y>u\}
$$

as $u \rightarrow \infty$. Hence

$$
\mathbb{P}\{Y>u-1\} \sim \mathbb{P}\{Y>u\}, \quad u \rightarrow \infty .
$$

Consequently, for any $\varepsilon \in(0,1)$ there exists $A>0$ such that for any $v>A$ we have $\mathbb{P}\{Y>v\} \geq(1-\varepsilon) \mathbb{P}\{Y>v-1\}$. Thus, for sufficiently large $u$

$\mathbb{P}\{Y>u\} \geq(1-\varepsilon) \mathbb{P}\{Y>u-1\} \geq(1-\varepsilon)^{2} \mathbb{P}\{Y>u-2\} \geq(1-\varepsilon)^{u-A} \mathbb{P}\{Y>A\}$ implying that for each $B>0$

$$
\lim _{u \rightarrow \infty} \frac{\exp (-B u)}{\mathbb{P}\{Y>u\}}=0
$$

and thus the claim follows. 


\subsection{Proof of Theorem 3.1}

i) We first give the upper bound. For any $\varepsilon \in\left(0, t_{0}\right), u>0$, we derive that

$$
\begin{aligned}
\mathbb{P}\left\{\sup _{t \in[0, \mathcal{T}]} X(t)>u\right\} \leq & \mathbb{P}\left\{\sup _{t \in[0, \mathcal{T}]} X(t)>u, \mathcal{T}<t_{0}-\varepsilon\right\} \\
& +\mathbb{P}\left\{\sup _{t \in[0, \mathcal{T}]} X(t)>u, \mathcal{T} \geq t_{0}-\varepsilon\right\} \\
\leq & \mathbb{P}\left\{\sup _{t \in\left[0, t_{0}-\varepsilon\right]} X(t)>u\right\} \\
& +\mathbb{P}\left\{\sup _{t \in[0, \infty)} X(t)>u\right\} \mathbb{P}\left\{\mathcal{T} \geq t_{0}-\varepsilon\right\},
\end{aligned}
$$

Similarly, for any $u>0$

$$
\begin{aligned}
\mathbb{P}\left\{\sup _{t \in[0, \mathcal{T}]} X(t)>u\right\} \geq & \mathbb{P}\left\{\sup _{t \in\left[0, t_{0}+\varepsilon\right]} X(t)>u\right\} \mathbb{P}\left\{\mathcal{T} \geq t_{0}+\varepsilon\right\} \\
\geq & \mathbb{P}\left\{\sup _{t \in[0, \infty)} X(t)>u\right\} \\
& \left.-\mathbb{P}\left\{\sup _{t \in\left[t_{0}+\varepsilon, \infty\right)} X(t)>u\right\}\right) \mathbb{P}\left\{\mathcal{T} \geq t_{0}+\varepsilon\right\} .
\end{aligned}
$$

Choosing $\varepsilon$ small enough such that limsup $\sigma(t)<\sigma\left(t_{0} \pm \varepsilon\right)<1$ and using the Borell-TIS inequality (e.g., Adler and Taylor 2007), we conclude that

$$
\mathbb{P}\left\{\sup _{t \in\left[0, t_{0}-\varepsilon\right]} X(t)>u\right\}=o\left(\mathbb{P}\left\{\sup _{t \in[0, \infty)} X(t)>u\right\}\right)
$$

and

$$
\mathbb{P}\left\{\sup _{t \in\left[t_{0}+\varepsilon, \infty\right)} X(t)>u\right\}=o\left(\mathbb{P}\left\{\sup _{t \in[0, \infty)} X(t)>u\right\}\right)
$$

as $u \rightarrow \infty$. Thus the claim of the first statement follows by letting $\varepsilon \rightarrow 0$. 
ii) Assume that $\mathcal{T}$ is $u^{1-2 /(\gamma(1+\beta))}$-insensitive and let $\varepsilon_{u}=u^{-2 /(1+\beta)}$. With similar arguments as in the proof of statement $i$ ) we obtain

$$
\begin{aligned}
p(X, \mathcal{T}, \gamma, u)= & \mathbb{P}\left\{\sup _{t \in\left[0, \mathcal{T} / u^{\gamma}\right]} X(t)>u\right\} \\
\leq & \mathbb{P}\left\{\sup _{t \in\left[0, t_{0}-\varepsilon_{u}\right]} X(t)>u\right\} \\
& +\mathbb{P}\left\{\sup _{t \in[0, \infty)} X(t)>u\right\} \mathbb{P}\left\{\mathcal{T}>\left(t_{0}-\varepsilon_{u}\right) u^{\gamma}\right\}
\end{aligned}
$$

and

$$
\begin{aligned}
p(X, \mathcal{T}, \gamma, u) \geq & \mathbb{P}\left\{\sup _{t \in\left[0, t_{0}+\varepsilon_{u}\right]} X(t)>u\right\} \mathbb{P}\left\{\mathcal{T}>\left(t_{0}+\varepsilon_{u}\right) u^{\gamma}\right\} \\
\geq & \mathbb{P}\left\{\sup _{t \in[0, \infty)} X(t)>u\right\} \\
& \left.-\mathbb{P}\left\{\sup _{t \in\left[t_{0}+\varepsilon_{u}, \infty\right)} X(t)>u\right\}\right) \mathbb{P}\left\{\mathcal{T}>\left(t_{0}+\varepsilon_{u}\right) u^{\gamma}\right\} .
\end{aligned}
$$

Further, by insensitivity of $\mathcal{T}$ as $u \rightarrow \infty$

$$
\mathbb{P}\left\{\mathcal{T}>\left(t_{0} \pm \varepsilon_{u}\right) u^{\gamma}\right\} \sim \mathbb{P}\left\{\mathcal{T}>t_{0} u^{\gamma}\right\}
$$

Thanks to Piterbarg inequality (cf. Theorem 8.1 of Piterbarg 1996), combined with A3, for all $u$ large we have

$$
\mathbb{P}\left\{\sup _{t \in\left[0, t_{0}-\varepsilon_{u}\right]} X(t)>u\right\} \leq \mathbb{Q} u^{2 / r-1} \exp \left(-\frac{u^{2}}{2}\right) \exp \left(-\frac{a u^{2}\left(\varepsilon_{u}\right)^{\beta}}{4}\right)
$$

for some positive constant $\mathbb{Q}$ not depending on $u$. Similarly, using additionally BorellTIS inequality, for $u$ large and some $G>t_{0}$

$$
\begin{aligned}
\mathbb{P}\left\{\sup _{t \in\left[t_{0}+\varepsilon_{u}, \infty\right)} X(t)>u\right\} \leq & \mathbb{P}\left\{\sup _{t \in\left[t_{0}+\varepsilon_{u}, G\right]} X(t)>u\right\}+\mathbb{P}\left\{\sup _{t \in[G, \infty)} X(t)>u\right\} \\
\leq & \mathbb{Q} u^{2 / r-1} \exp \left(-\frac{u^{2}}{2}\right) \exp \left(-\frac{a u^{2}\left(\varepsilon_{u}\right)^{\beta}}{4}\right) \\
& +\exp \left(-\frac{\left.\left(u-\mathbb{E}\left(\sup _{t \in[G, \infty)} X(t)\right)\right)^{2}\right)}{2 \sup _{t \in[G, \infty)} \sigma^{2}(t)}\right)
\end{aligned}
$$


Hence, using that $\varepsilon_{u}=u^{-2 /(1+\beta)}$ and the results of Lemma 5.1

$$
\lim _{u \rightarrow \infty} \frac{\exp \left(-\frac{a}{4} u^{\frac{2}{1+\beta}}\right)}{\mathbb{P}\left\{\mathcal{T}>t_{0} u^{\gamma}\right\}}=0
$$

and thus

$$
\begin{aligned}
& \mathbb{P}\left\{\sup _{t \in\left[t_{0}+\varepsilon_{u}, \infty\right)} X(t)>u\right\}=o\left(\mathbb{P}\left\{\sup _{t \in[0, \infty)} X(t)>u\right\}\right), \\
& \mathbb{P}\left\{\sup _{t \in\left[0, t_{0}-\varepsilon_{u}\right]} X(t)>u\right\}=o\left(\mathbb{P}\left\{\sup _{t \in[0, \infty)} X(t)>u\right\} \mathbb{P}\left\{\mathcal{T}>\left(t_{0}-\varepsilon_{u}\right) u^{\gamma}\right\}\right) .
\end{aligned}
$$

Consequently, combining Eqs. 29-32 we obtain

$$
p(X, \mathcal{T}, \gamma, u) \sim \mathbb{P}\left\{\sup _{t \in[0, \infty)} X(t)>u\right\} \mathbb{P}\left\{\mathcal{T}>t_{0} u^{\gamma}\right\}
$$

as $u \rightarrow \infty$, which completes the proof.

\subsection{Proof of Theorem 3.3}

For the proof we need to distinguish between three different cases depending on the value of $\gamma p$. For notational simplicity, let $T_{u}:=\mathcal{T} / u^{\gamma}$.

Case $\gamma p<2$. For some $\varepsilon>0$ small enough and $u$ large

$$
\begin{aligned}
p(X, \mathcal{T}, \gamma, u)= & \mathbb{P}\left\{\sup _{t \in\left[0, T_{u}\right]} X(t)>u, T_{u}>u^{\frac{2-\gamma p}{p}-\varepsilon}\right\} \\
& +\mathbb{P}\left\{\sup _{t \in\left[0, T_{u}\right]} X(t)>u, T_{u} \leq u^{\frac{2-\gamma p}{p}-\varepsilon}\right\} \\
\geq & \mathbb{P}\left\{\mathcal{T}>u^{\gamma+\frac{2-\gamma p}{p}-\varepsilon}\right\} \mathbb{P}\left\{\begin{array}{c}
\left.\sup _{\frac{2-\gamma p}{p}-\varepsilon} X(t)>u\right\} \\
\geq \in\left[0, u^{\frac{2-\gamma p}{p}}-\varepsilon\right.
\end{array}\right\} \mathbb{P}\left\{X\left(t_{0}\right)>u\right\} \\
\geq & \mathbb{P}\left\{\mathcal{T}>u^{\gamma+\frac{2-\gamma p}{p}}\right\} \\
& \stackrel{\log }{\sim} \mathbb{P}\left\{X\left(t_{0}\right)>u\right\},
\end{aligned}
$$

which follows from the fact that, by the assumption on $\mathcal{T}, \mathbb{P}\left\{\mathcal{T}>u^{\gamma+\frac{2-\gamma p}{p}-\varepsilon}\right\} \stackrel{\log }{\sim}$ $\exp \left(-L u^{2-\varepsilon p}\right)$, while $\mathbb{P}\left\{X\left(t_{0}\right)>u\right\} \stackrel{\log }{\sim} \exp \left(-u^{2} / 2\right)$. Since, in view of Theorem 2.1

$$
p(X, \mathcal{T}, \gamma, u) \leq \mathbb{P}\left\{\sup _{t \in[0, \infty)} X(t)>u\right\} \stackrel{\log }{\sim} \mathbb{P}\left\{X\left(t_{0}\right)>u\right\},
$$

then the claim (15) follows. 
Case $\gamma p=2$. Let $\widehat{\sigma}(s), \widetilde{\sigma}_{L, \gamma}(s), s \geq 0$ and $A_{0}$ be defined as in Eqs. 13 and 14 respectively. The lower bound follows from the fact that

$$
\begin{aligned}
p(X, \mathcal{T}, \gamma, u) & =\mathbb{P}\left\{\sup _{t \in\left[0, T_{u}\right]} X(t)>u, T_{u}>A_{0}\right\}+\mathbb{P}\left\{\sup _{t \in\left[0, T_{u}\right]} X(t)>u, T_{u} \leq A_{0}\right\} \\
& \geq \mathbb{P}\left\{\mathcal{T}>A_{0} u^{\gamma}\right\} \mathbb{P}\left\{\sigma\left(A_{0}\right) \mathcal{N}>u\right\} \\
& \stackrel{\log }{\sim} \exp \left(-L A_{0}^{2 / \gamma} u^{2}\right) \exp \left(-\frac{u^{2}}{2 \sigma^{2}\left(A_{0}\right)}\right) \\
& =\exp \left(-\widetilde{\sigma}_{L, \gamma}\left(A_{0}\right) u^{2}\right),
\end{aligned}
$$

where $\mathcal{N}$ is a standard Gaussian (i.e., an $N(0,1))$ random variable.

Next we derive an upper bound. For some $0<m<1<M$ (to be determined later) we have

$$
\begin{aligned}
p(X, \mathcal{T}, \gamma, u)=-\int_{0}^{\infty} \mathbb{P}\left\{\sup _{t \in[0, s]} X(t)>u\right\} d \mathbb{P}\left\{T_{u}>s\right\} \\
=-\int_{0}^{m A_{0}} \mathbb{P}\left\{\sup _{t \in[0, s]} X(t)>u\right\} d \mathbb{P}\left\{T_{u}>s\right\} \\
\quad-\int_{m A_{0}}^{M A_{0}} \mathbb{P}\left\{\sup _{t \in[0, s]} X(t)>u\right\} d \mathbb{P}\left\{T_{u}>s\right\} \\
-\int_{M A_{0}}^{\infty} \mathbb{P}\left\{\sup _{t \in[0, s]} X(t)>u\right\} d \mathbb{P}\left\{T_{u}>s\right\} \\
:=I_{1}(u)+I_{2}(u)+I_{3}(u) .
\end{aligned}
$$

We analyze $I_{i}(u), i=1,2,3$ separately.

Ad. $I_{2}(u)$. Using Piterbarg inequality and integration by parts, we have that

$$
\begin{aligned}
I_{2}(u) \leq & -\int_{m A_{0}}^{M A_{0}}\left(\mathbb{Q} M A_{0} u^{2 / r} \Psi\left(\frac{u}{\widehat{\sigma}(s)}\right)\right) d \mathbb{P}\left\{T_{u}>s\right\} \\
= & -\mathbb{Q} M A_{0} u^{2 / r}\left(\Psi\left(\frac{u}{\widehat{\sigma}\left(M A_{0}\right)}\right) \mathbb{P}\left\{\mathcal{T}>M A_{0} u^{\gamma}\right\}\right. \\
& -\Psi\left(\frac{u}{\widehat{\sigma}\left(m A_{0}\right)}\right) \mathbb{P}\left\{\mathcal{T}>m A_{0} u^{\gamma}\right\} \\
+ & \left.\int_{m A_{0}}^{M A_{0}} \frac{u}{\sqrt{2 \pi}}\left(\frac{1}{\widehat{\sigma}(s)}\right)^{\prime} \exp \left(-\frac{u^{2}}{2 \widehat{\sigma}^{2}(s)}\right) \mathbb{P}\left\{T_{u}>s\right\} d s\right) \\
:= & I_{2,1}(u)+I_{2,2}(u)+I_{2,3}(u) \leq I_{2,2}(u)+I_{2,3}(u) .
\end{aligned}
$$

Next we find bounds for $I_{2, i}(u), i=2,3$ one by one. It straightforwardly follows that

$$
I_{2,2}(u) \stackrel{\log }{\sim} \exp \left(-\left(\frac{1}{2 \widehat{\sigma}^{2}\left(m A_{0}\right)}+L\left(m A_{0}\right)^{2 / \gamma}\right) u^{2}\right)
$$

and, for any $\varepsilon_{2}>0$ and $u$ large 


$$
\begin{aligned}
I_{2,3}(u) & \leq \mathbb{Q} M A_{0} \frac{u^{2 / r+1}}{\sqrt{2 \pi}} \max _{s \in\left[m A_{0}, M A_{0}\right]}\left(\frac{-1}{\widehat{\sigma}(s)}\right)^{\prime} \int_{m A_{0}}^{M A_{0}} \exp \left(-\left(\frac{1}{2 \widehat{\sigma}^{2}(s)}+L\left(1-\varepsilon_{2}\right) s^{2 / \gamma}\right) u^{2}\right) d s \\
& \leq \mathbb{Q}\left(M A_{0}\right)^{2} \frac{u^{2 / r+1}}{\sqrt{2 \pi}} \max _{s \in\left[m A_{0}, M A_{0}\right]}\left(\frac{-1}{\widehat{\sigma}(s)}\right)^{\prime} \exp \left(-\inf _{s \in\left[m A_{0}, M A_{0}\right]}\left(\frac{1}{2 \widehat{\sigma}^{2}(s)}+L\left(1-\varepsilon_{2}\right) s^{2 / \gamma}\right) u^{2}\right) .
\end{aligned}
$$

Consequently, letting $\varepsilon_{2} \rightarrow 0$ we obtain

$$
\limsup _{u \rightarrow \infty} \frac{\log I_{2,3}(u)}{u^{2}} \leq-\widetilde{\sigma}_{L, \gamma}\left(A_{0}\right) .
$$

Ad. $I_{1}(u)$. By the Piterbarg inequality we have that

$$
I_{1}(u) \leq \mathbb{P}\left\{\sup _{t \in\left[0, m A_{0}\right]} X(t)>u\right\} \leq \mathbb{Q} u^{2 / r+1} \exp \left(-\frac{u^{2}}{2 \widehat{\sigma}^{2}\left(m A_{0}\right)}\right),
$$

where $r \in(0,2]$ is as in $\mathbf{A 3}$.

Ad. $I_{3}(u)$. We straightforwardly have that

$$
I_{3}(u) \leq \mathbb{P}\left\{T_{u}>M A_{0}\right\} \stackrel{\log }{\sim} \exp \left(-L\left(M A_{0}\right)^{2 / \gamma} u^{2}\right) .
$$

Now we are ready to determine both constants $m$ and $M$. First, choose $m$ such that

$$
\frac{1}{2 \widehat{\sigma}^{2}\left(m A_{0}\right)}>\widetilde{\sigma}_{L, \gamma}\left(A_{0}\right)
$$

and then choose $M$ such that

$$
L\left(M A_{0}\right)^{2 / \gamma}>\widetilde{\sigma}_{L, \gamma}\left(A_{0}\right) .
$$

We conclude from Eqs. 34-37 that

$$
\limsup _{u \rightarrow \infty} \frac{\log p(X, \mathcal{T}, \gamma, u)}{u^{2}} \leq-\widetilde{\sigma}_{L, \gamma}\left(A_{0}\right)
$$

Consequently, combination of Eq. 33 with Eq. 38 leads to

$$
\lim _{u \rightarrow \infty} \frac{\log p(X, \mathcal{T}, \gamma, u)}{u^{2}}=-\widetilde{\sigma}_{L, \gamma}\left(A_{0}\right)
$$

$\underline{\text { Case } \gamma p>2 \text { and } \sigma(0)>0}$. Let $\varepsilon \in\left(0, \frac{\gamma p-2}{p}\right)$. Then we have $\left(\operatorname{set} u_{\varepsilon}:=u^{\frac{2-\gamma p}{p}+\varepsilon}\right)$

$$
\begin{aligned}
p(X, \mathcal{T}, \gamma, u)= & \mathbb{P}\left\{\sup _{t \in\left[0, \mathcal{T}_{u}\right]} X(t)>u, \mathcal{T}_{u}>u_{\varepsilon}\right\} \\
& +\mathbb{P}\left\{\sup _{t \in\left[0, \mathcal{T}_{u}\right]} X(t)>u, T_{u} \leq u_{\varepsilon}\right\} \\
\leq & \mathbb{P}\left\{\mathcal{T}>u^{\frac{2}{p}+\varepsilon}\right\}+\mathbb{P}\left\{\sup _{t \in\left[0, u_{\varepsilon}\right]} X(t)>u\right\} .
\end{aligned}
$$


Since $-\log \mathbb{P}\{\mathcal{T}>u\}$ is regularly varying at infinity with index $p$, then

$$
\lim _{u \rightarrow \infty} \frac{\log \mathbb{P}\left\{\mathcal{T}>u^{\frac{2}{p}+\varepsilon}\right\}}{u^{2}}=-\infty
$$

and, by Borell-TIS inequality, for sufficiently large $u$

$$
\mathbb{P}\left\{\sup _{t \in\left[0, u_{\varepsilon}\right]} X(t)>u\right\} \leq 2 \exp \left(-\frac{\left(u-\mathbb{E}\left(\sup _{t \in\left[0, u_{\varepsilon}\right]} X(t)\right)\right)^{2}}{2 \max _{t \in\left[0, u_{\varepsilon}\right]} \sigma^{2}(t)}\right) .
$$

Combining the above with

$$
p(X, \mathcal{T}, \gamma, u) \geq \mathbb{P}\{X(0)>u\}
$$

we obtain that

$$
\lim _{u \rightarrow \infty} \frac{\log p(X, \mathcal{T}, \gamma, u)}{u^{2}}=-\frac{1}{2 \sigma^{2}(0)}
$$

which proves (17).

Case $\gamma p>2$ and $\sigma(t)=D t^{\eta}(1+o(1))$ as $t \downarrow 0$. Let $g(t)=\frac{1}{2 D^{2} t^{2 \eta}}+L t^{p}, t \geq 0$, which has a unique minimum point at $t^{*}=\left(\frac{\eta}{p L D^{2}}\right)^{1 /(2 \eta+p)}$ with

$$
A_{1}:=g\left(t^{*}\right)=\frac{1}{2} D^{-\frac{2 p}{2 \eta+p}}(L p / \eta)^{\frac{2 \eta}{2 \eta+p}}+L^{\frac{2 \eta}{2 \eta+p}}\left(\eta /\left(p D^{2}\right)\right)^{\frac{p}{2 \eta+p}} .
$$

Setting $\mu=\frac{p \gamma-2}{2 \eta+p}>0$ we may write

$$
\begin{aligned}
p(X, \mathcal{T}, \gamma, u)= & \mathbb{P}\left\{\sup _{t \in\left[0, T_{u}\right]} X(t)>u, T_{u}>t^{*} u^{-\mu}\right\} \\
& +\mathbb{P}\left\{\sup _{t \in\left[0, T_{u}\right]} X(t)>u, T_{u} \leq t^{*} u^{-\mu}\right\} \\
\geq & \mathbb{P}\left\{\sup _{t \in\left[0, t^{*} u^{-\mu}\right]} X(t)>u\right\} \mathbb{P}\left\{\mathcal{T}>t^{*} u^{\gamma-\mu}\right\} \\
\geq & \mathbb{P}\left\{X\left(t^{*} u^{-\mu}\right)>u\right\} \mathbb{P}\left\{\mathcal{T}>t^{*} u^{\gamma-\mu}\right\} \\
& \stackrel{\log }{\sim} \exp \left(-\frac{u^{2+2 \eta \mu}}{2 D^{2}\left(t^{*}\right)^{2 \eta}}-L\left(t^{*}\right)^{p} u^{p(\gamma-\mu)}\right) \\
= & \exp \left(-A_{1} u^{\frac{2 \eta \gamma p+2 p}{2 \eta+p}}\right) .
\end{aligned}
$$

In order to derive an upper bound, we replace $A_{0}$ with $t^{*} u^{-\mu}$ in the upper estimate of the case $\gamma p=2$. Following step-by-step the same argument as in the upper bound 
of the case $\gamma p=2$, we conclude that

$$
\limsup _{u \rightarrow \infty} \frac{\log p(X, \mathcal{T}, \gamma, u)}{u^{\frac{2 \eta \gamma p+2 p}{2 \eta+p}}} \leq-A_{1}
$$

and thus the proof is complete.

\subsection{Proof of Propositions 4.1 and 4.2}

By the self-similar property of the $\mathrm{fBm}$ we see that

$$
\mathbb{P}\left\{\sup _{t \in[0, T]}\left(B_{\alpha}(Y(t))-c(Y(t))^{\theta}\right)>u\right\}=\mathbb{P}\left\{\sup _{t \in\left[0, Y(T) / u^{1 / \theta}\right]} \frac{B_{\alpha}(t)}{1+c t^{\theta}}>u^{1-\frac{\alpha}{2 \theta}}\right\}
$$

The function $V(t)=\frac{t^{\alpha / 2}}{1+c t^{\theta}}$ attains its maximum at the unique point

$$
t_{0}=\left(\frac{\alpha / 2}{c(\theta-\alpha / 2)}\right)^{1 / \theta}
$$

and

$$
V_{0}=V\left(t_{0}\right)=\frac{2 \theta-\alpha}{2 \theta}\left(\frac{\alpha}{c(2 \theta-\alpha)}\right)^{\frac{\alpha}{2 \theta}} .
$$

Re-writing (39), we have

$$
\begin{aligned}
& \mathbb{P}\left\{\sup _{t \in[0, T]}\left(B_{\alpha}(Y(t))-c(Y(t))^{\theta}\right)>u\right\}=\mathbb{P}\left\{\sup _{t \in\left[0, Y(T) / u^{1 / \theta}\right]} Z(t)>V_{0}^{-1} u^{1-\frac{\alpha}{2 \theta}}\right\} \\
& =\mathbb{P}\left\{\begin{array}{c}
\sup _{t \in\left[0, \frac{Y(T)\left(V_{0}\right)-\frac{2}{2 \theta-\alpha}}{(v(u)) \frac{2}{2 \theta-\alpha}}\right]} Z(t)>v(u) \\
{[t}
\end{array}\right\},
\end{aligned}
$$

where

$$
Z(t)=\frac{B_{\alpha}(t)}{t^{\alpha / 2}} \frac{V(t)}{V_{0}}, \quad t \geq 0 \text { and } v(u)=V_{0}^{-1} u^{1-\frac{\alpha}{2 \theta}} .
$$

It is straightforward to check that

$$
\sqrt{\mathbb{E}\left(Z^{2}(t)\right)}=1-\frac{1}{8} c^{\frac{2}{\theta}} \alpha^{1-\frac{2}{\theta}}(2 \theta-\alpha)^{1+\frac{2}{\theta}}\left(t-t_{0}\right)^{2}+o\left(\left(t-t_{0}\right)^{2}\right)
$$

as $t \rightarrow t_{0}$ and

$$
\operatorname{Cov}\left(\frac{Z(t)}{\sqrt{\mathbb{E}\left(Z^{2}(t)\right)}}, \frac{Z(s)}{\sqrt{\mathbb{E}\left(Z^{2}(s)\right)}}\right)=1-\frac{1}{2 t_{0}^{\alpha}}|t-s|^{\alpha}+o\left(|t-s|^{\alpha}\right)
$$


as $s, t \rightarrow t_{0}$. Moreover, for any given positive constant $H$, it is derived that, for $t, s \in[0, H]$ with $|t-s|<1$

$$
\begin{aligned}
\mathbb{E}(Z(t)-Z(s))^{2} & =V_{0}^{-2} \mathbb{E}\left(\left(\frac{B_{\alpha}(t)}{1+c t^{\theta}}-\frac{B_{\alpha}(s)}{1+c t^{\theta}}\right)+\left(\frac{B_{\alpha}(s)}{1+c t^{\theta}}-\frac{B_{\alpha}(s)}{1+c s^{\theta}}\right)\right)^{2} \\
& \leq 2\left(V_{0}\right)^{-2}\left(\mathbb{E}\left(\frac{B_{\alpha}(t)}{1+c t^{\theta}}-\frac{B_{\alpha}(s)}{1+c t^{\theta}}\right)^{2}+\mathbb{E}\left(\frac{B_{\alpha}(s)}{1+c t^{\theta}}-\frac{B_{\alpha}(s)}{1+c s^{\theta}}\right)^{2}\right) \\
& =2\left(V_{0}\right)^{-2}\left(\frac{|t-s|^{\alpha}}{\left(1+c t^{\theta}\right)^{2}}+s^{\alpha}\left(\frac{1}{1+c t^{\theta}}-\frac{1}{1+c s^{\theta}}\right)^{2}\right) \\
& \leq \mathbb{Q}|t-s|^{\alpha} .
\end{aligned}
$$

Thus assumptions A1-A4 are satisfied. Additionally, by Theorem 1 in Hüsler and Piterbarg (1999)

$\mathbb{P}\left\{\sup _{t \in[0, \infty)} Z(t)>v(u)\right\} \sim Q \mathcal{H}_{\alpha} u^{\frac{(2 \theta-\alpha)(2-\alpha)}{2 \theta \alpha}} \Psi\left(\frac{2 \theta}{2 \theta-\alpha}\left(\frac{\alpha}{c(2 \theta-\alpha)}\right)^{-\frac{\alpha}{2 \theta}} u^{\frac{2 \theta-\alpha}{2 \theta}}\right), \quad u \rightarrow \infty$

where $Q:=2^{\frac{1}{2}+\frac{1}{\alpha}} \sqrt{\pi} c^{\frac{2-\alpha}{2 \theta}} \alpha^{\frac{\alpha-2-\theta}{2 \theta}} \theta^{\frac{2-\alpha}{\alpha}}(2 \theta-\alpha)^{\frac{\theta \alpha-4 \theta+2 \alpha-\alpha^{2}}{2 \theta \alpha}}$.

The rest of the proof follows from an application of Theorem 3.1 statement ii) and Theorem 3.3.

\subsection{Proof of Propositions 4.3 and 4.4}

Note that, for each $u>0$, using notation introduced in Section 5.3

$$
\mathbb{P}\left\{\sup _{t \in[0, T]} B_{\alpha}(Y(t))-c(Y(t))^{\theta}>u\right\} \leq \mathbb{P}\left\{\sup _{t \in\left[0, Y(T) / u^{1 / \theta}\right]} Z(t)>V_{0}^{-1} u^{1-\frac{\alpha}{2 \theta}}\right\}
$$

Thus it suffices to find logarithmically tight lower bounds for each subclass of densities of $Y(T)$. The idea of the proof is the same both for the density of $Y(T)$ being regularly varying and log-power tailed and heavily uses the idea of getting the lower bound in the proof of Theorem 3.3. Hence we give only the argument for $Y(T)$ having regularly varying density function with index $\lambda+1$. Under this scenario

$$
\begin{aligned}
& \mathbb{P}\left\{\sup _{t \in[0, T]}\left(B_{\alpha}(Y(t))-c(Y(t))^{\theta}\right)>u\right\} \geq \mathbb{P}\left\{\frac{B_{\alpha}(Y(T))}{u+c(Y(T))^{\theta}}>1\right\} \\
\geq & \min _{t \in\left[t_{0} u^{1 / \theta}-u^{1 /(2 \theta)}, t_{0} u^{1 / \theta}+u^{1 /(2 \theta)}\right]} \mathbb{P}\left\{\frac{B_{\alpha}(t)}{u+c t^{\theta}}>1\right\} \\
& X \mathbb{P}\left\{Y(T) \in\left[t_{0} u^{1 / \theta}-u^{1 /(2 \theta)}, t_{0} u^{1 / \theta}+u^{1 /(2 \theta)}\right]\right\} .
\end{aligned}
$$


Using that $\mathbb{P}\left\{Y(T) \in\left[t_{0} u^{1 / \theta}-u^{1 /(2 \theta)}, t_{0} u^{1 / \theta}+u^{1 /(2 \theta)}\right]\right\}$ is regularly varying at $\infty$ and

$$
\lim _{u \rightarrow \infty} u^{\frac{\alpha}{\theta}-2} \log \left(\min _{t \in\left[t_{0} u^{1 / \theta}-u^{1 /(2 \theta)}, t_{0} u^{1 / \theta}+u^{1 /(2 \theta)}\right]} \mathbb{P}\left\{\frac{B_{\alpha}(t)}{u+c t^{\theta}}>1\right\}\right)=-\frac{1}{2 V_{0}^{2}}
$$

we obtain a logarithmically tight lower bound, and thus the proof is complete.

Acknowledgement We are thankful to the referees for several comments and suggestions. K. Dębicki was partially supported by NCN Grant No 2011/01/B/ST1/01521 (2011-2013). K. Dębicki, E. Hashorva and L. Ji kindly acknowledge partial support by the Swiss National Science Foundation Grant 200021140633/1 and by the project RARE -318984, a Marie Curie FP7 IRSES Fellowship.

\section{References}

Adler, R.J.: An Introduction to Continuity, Extrema, and Related Topics for General Gaussian Processes, Inst. Math. Statist. Lecture Notes Monogr. Ser. 12, Inst. Math. Statist, Hayward, CA (1990)

Adler, R.J., Taylor, J.E.: Random Fields and Geometry. Springer (2007)

Arendarczyk, M., Dębicki, K.: Asymptotics of supremum distribution of a Gaussian process over a Weibullian time. Bernoulli 17, 194-210 (2011)

Arendarczyk, M., Dębicki, K.: Exact asymptotics of supremum of a stationary Gaussian process over a random interval. Stat. Prob. Lett. 82, 645-652 (2012)

Dębicki, K., Rolski, T.: A note on transient Gaussian fluid models. Queueing Systems 41, 321-342 (2002)

Dȩbicki, K., van Uitert, M.: Large buffer asymptotics for generalized processor sharing queues with Gaussian inputs. Queueing Syst. 54, 111-120 (2006)

Foss, S., Korshunov, D., Zachary, S.: An introduction to Heavy-tailed and Subexponential Distributions, 2nd edn. Springer-Verlag, New York (2013)

Fotopoulos, S., Luo, Y.: Subordinated Gaussian Processes, the Log-Return Principles. Washington State University, Washington (2011)

Geman, H., Madan, D.B., Yor, M.: Time changes for Lévy processes. Math. Financ. 11, 79-96 (2001)

Hashorva, E., Ji, L., Piterbarg, V.I.: On the supremum of gamma-reflected processes with fractional Brownian motion as input. Stoch. Proc. Appl. 123, 4111-4127 (2013)

Hüsler, J., Piterbarg, V.: Extremes of a certain class of Gaussian processes. Stochastic Process. Appl. 83, 257-271 (1999)

Kozubowski, T.J., Meerschaert, M.M., Molz, F.J., Lu, S.: Fractional Laplace model for hydraulic conductivity. Geophhys. Res. Lett. 31, 1-4 (2004)

Kozubowski, T.J., Meerschaert, M.M., Podgórski, K.: Fractional Laplace motion. Adv. Appl. Probab. 38, 451-464 (2006)

Michna, Z.: Self-similar processes in collective risk theory. J. Appl. Math. Stoch. Anal. 11, 429-448 (1998)

Palmowski, Z., Zwart, B.: Tail asymptotics of the supremum of a regenerative process. J. Appl. Prob. 44, 349-365 (2007)

Piterbarg, V.I.: Asymptotic Methods in the Theory of Gaussian Processes and Fields. In: Transl. Math. Monogr., vol. 148. AMS, Providence (1996)

Resnick, S.I.: Extreme Values (1987). Regular Variation, and Point Processes. Springer

Tan, Z., Hashorva, E.: Exact tail asymptotics of the supremum of strongly dependent gaussian processes over a random interval. Lith. Math. J 53, 91-102 (2013)

Wu, R., Wang, W.: The hitting time for a Cox risk process. J. Comp. Appl. Math 236, 2706-2716 (2012)

Zwart, B., Borst, S., Dȩbicki, K.: Subexponential asymptotics of hybrid fluid and ruin models. Ann. Appl. Probab. 15, 500-517 (2005) 